\title{
Unifying Multi-Radio Communication Technologies to Enable mMTC Applications in B5G Networks
}

\author{
Radek Mozny ${ }^{1}$, Martin Stusek ${ }^{1}$, Pavel Masek ${ }^{1}$, Konstantin Mikhaylov ${ }^{1,2}$, and Jiri Hosek ${ }^{1}$ \\ ${ }^{1}$ Department of Telecommunications, Brno University of Technology, Brno, Czech Republic \\ ${ }^{2}$ Center for Wireless Communications, University of Oulu, Oulu, Finland \\ $\triangle$ Contact author's e-mail: xmozny03@vutbr.cz
}

\begin{abstract}
Even though the wireless communication technologies have evolved significantly in the last decade, the performance requirements of versatile massive Machine-Type Communication (mMTC) use-cases grow at even faster pace. For this reason, the challenge of providing energy-efficient, reliable, secure, and variable devices with ubiquitous connectivity cannot be addressed by any single Radio Access Technology (RAT) available today. Therefore, the concept of multi-RAT devices in the context of next-generation communication technologies (5G and beyond 5G (B5G)) comes into play. In this paper, we investigate the utility of multi-RAT IoT connectivity in practice with respect to a Smart City scenario, in which the location of public transport vehicles e.g., trams and buses is tracked and reported by a device equipped with two Low-Power Wide-Area Network (LPWAN) technologies i.e., Narrowband IoT (3rd Generation Partnership Project (3GPP)) and LoRaWAN (non-3GPP). Both technologies got matured and have shown their momentum in the recent years, and are expected to play the key role also in the future. We first detail the constructed multi-RAT prototype and then report the initial results of its evaluation in a pilot deployment in the city of Brno, Czech Republic. The obtained results illustratively confirm the feasibility of joint usage of two diverse LPWAN RATs while their combination brings higher flexibility, reliability, and improve the overall Quality of Service (QoS) to wireless connectivity.
\end{abstract}

\section{INTRODUCTION}

During the last decade, the concept of the Internet of Things (IoT) was maturing towards enabling ubiquitous connectivity for the machines. Challenged by the ever-increasing amount of data transmitted over the wireless connections and by the never-stopping grow of the demands for lowering the price and improving the operational parameters, it is more than clear that legacy cellular systems cannot fulfill all these requirements even in theory [1]. The Smart City scenario is especially challenging in this context due to the sheer variety of applications and services to be provided. At the very same time, this environment already features a number of the legacy connectivity technologies deployed [2].

The question is set to whether the next-generation (heterogeneous) systems known as $5 \mathrm{G}$ can act as the enablers for the new communication use-cases in the massive Machinetype Communication (mMTC) scenarios impossible to realize with traditional communication technologies, i.e., 2G/3G/4G. These technologies are inherently designed and optimized for Human-to-Human $(\mathrm{H} 2 \mathrm{H})$ communication, thus having some drawbacks for Machine-to-Machine (M2M) traffic. Here, the Low-Power Wide-Area Network (LPWAN) technologies pri- marily targeted for mMTC have entered the game. As the situation stands right now, the majority of the available LPWAN technologies e.g., LoRaWAN, Sigfox, IEEE 802.11ah, and IEEE802.11p represent the non-3GPP Radio Access Technologies (RATs). To open the door for the interoperability between the 3GPP (Narrowband IoT and LTE Cat-M1) and non-3GPP systems, the Non-3GPP InterWorking Function (N3IWF) was introduced, enabling access 5G Core Network (CN) [3].

Since each LPWAN technology on the market offers a unique set of features, the idea of combining them in the course of one multifunctional device (sensor, actuator, aggregation gateway, etc.) paves the way to address the needs of the new-generation IoT devices, which will operate in the emerging 5G and beyond networks (B5G) [4]. Noticeably, the idea of soldering multiple radio access technology (RAT) transceivers on a single board is not new [5]. Already today, several chipset manufacturers do sell their solutions, offering short and long-range communication capability [6], [7]. Nevertheless, the current chipsets do not provide the possibility to combine recent LPWAN technologies (3GPP and non3GPP) in one chip.

\section{A. Our Contribution}

In this paper, we explore the idea of a multi-radio device for mMTC use-cases while overcoming the known limitations of individual LPWAN technologies e.g., the diverse characteristics of the frequency spectrum, restricted transmission power, limited duty-cycle (DC), or the interference caused by the other communication technologies competing for the scarce resources of the Industrial, Scientific and Medical (ISM) frequency band. The main contribution of this paper is condensed in what follows:

- We investigate the key benefits and drawbacks of two LPWAN technologies selected in the course of this work, i.e., LoRaWAN (non-3GPP) and Narrowband IoT (NBIoT) (3GPP).

- We thoughtfully describe our practical implementation of a multi-RAT prototype, the lessons learned as well as the results of its evaluation in a Smart City scenario for tracking the public transport vehicles.

- Based on our outcomes, we deliver an insight into how the two different LPWAN technologies can be combined and how such combinations can be used. These observations are especially important for the future B5G 
networks since they give insight into the possible role of the different RATs e.g., based on the frequency spectrum utilization or dynamically changing interferences and provide a new method to address the challenging requirements of the IoT applications.

\section{LPWAN IN THE CONTEXT OF MR-MMTC}

LPWAN technologies represent an efficient way to address the needs of mMTC applications. By their nature, the LPWAN technologies are optimized for infrequent transmissions of limited data size. This allows the IoT devices to expect the multi-year lifetime when powered by battery since most of the time a device can spend in a deep sleep mode with power consumption in the order of units of $\mu \mathrm{A}$ [8]. However, albeit sharing the common goal, the technical solutions of the different LPWAN RAT vary. To give a practical example, in Table I,we listed the key performance characteristics of the two LPWAN technologies dominating the market today - the NB-IoT and LoRaWAN. In the following sections, we first discuss their technical solution in more details and then justify NB-IoT - LoRaWAN multi-RAT combination.

\section{A. Narrowband IoT}

The NB-IoT operates in a narrow $180 \mathrm{kHz}$-wide band within the frequency spectrum licensed to telecom operators. This provides the flexibility to deploy NB-IoT on top of the existing Long Term Evolution (LTE) infrastructure via a software update. The NB-IoT reuses a significant part of LTE numerology reflected in the bandwidth corresponding to one Physical Resource Block (PRB) of its predecessor. Further, the NBIoT also utilizes Single Carrier Frequency Division Multiple Access (SC-FDMA) in the uplink channel and Orthogonal FDMA (OFDMA) in the downlink [8]. The design of NBIoT protocol is based on LTE and its procedures, which were substantially simplified, but the signaling still remain rather power-hungry.

The extended coverage of $+20 \mathrm{~dB}$ in comparison with traditional LTE, is ensured by the utilization of robust Modulation and Coding Schemes (MCS) which are further complemented by frame repetitions, and represent a crucial mechanism to increase the coverage. The energy efficiency is ensured primarily through the Power Saving Mode (PSM) and extended Discontinuous Reception (eDRX) mechanisms [9], which allow the device to enter deep-sleep mode, while still staying registered to the network and increase the period for paging reception, respectively. However, when connecting to the network or transmitting the data, the consumption of the NB-IoT radio grows owing to extensive signalling and high (up to $23 \mathrm{dBm}$ ) transmit power resulting in consumption of significant current (can reach $250 \mathrm{~mA}$ in peak). For this reason, over the past few years, many optimization and new functionalities have been added to NB-IoT (including e.g., the early data transmission (EDT) mechanism in Rel. 15).

\section{B. LoRaWAN}

The LoRaWAN technology in the European region utilizes the ISM spectrum shared with a plethora of other
TABLE I

KEY PARAMETERS OF LORAWAN AND NB-IOT TECHNOLOGIES.

\begin{tabular}{|c|c|c|}
\hline & LoRaWAN & NB-IoT \\
\hline Coverage (MCL) & $157 \mathrm{~dB}$ & $164 \mathrm{~dB}$ \\
\hline Technology & $\begin{array}{l}\text { PHY - Proprietary } \\
\text { MAC - Open }\end{array}$ & LTE \\
\hline Spectrum & Unlicensed & Licensed \\
\hline DC restrictions & Yes & No \\
\hline Max. ERP & $14 \mathrm{dBm}$ & $23 \mathrm{dBm}$ \\
\hline Modulation & $\begin{array}{l}\text { LoRa, } \\
\text { FSK }\end{array}$ & $\begin{array}{c}\pi / 2 \text { - BPSK, } \pi / 4 \text { - QPSK, } \\
\text { QPSK }(\mathrm{DL}+\mathrm{MT})\end{array}$ \\
\hline Downlink data rate & $\begin{array}{c}0.25-11 \mathrm{kbps}(\mathrm{LoRa}) \\
50 \mathrm{kbps}(\mathrm{FSK}) \\
\end{array}$ & $0.5-27.2 \mathrm{kbps}$ \\
\hline Uplink data rate & $\begin{array}{c}0.25-11 \mathrm{kbps}(\mathrm{LoRa}) \\
50 \mathrm{kbps}(\mathrm{FSK})\end{array}$ & $0.3-62.5 \mathrm{kbps}$ \\
\hline Max. payload UL & $242 \mathrm{~B}$ & $1600 \mathrm{~B}^{1}$ \\
\hline Max. payload DL & $242 B$ & $1600 \mathrm{~B}^{1}$ \\
\hline Bandwidth & $125,250 \mathrm{kHz}$ & $180 \mathrm{kHz}$ \\
\hline Duplex mode & half duplex & half duplex \\
\hline Random access & pure ALOHA & $\begin{array}{c}\text { pure ALOHA } \\
\text { slotted ALOHA (Class B) }\end{array}$ \\
\hline TX current & $45 \mathrm{~mA}$ & $220 \mathrm{~mA}$ \\
\hline Sleep current & $<2 \mu \mathrm{A}$ & $<3 \mu \mathrm{A}$ \\
\hline Battery life & $10+$ years & $10+$ years \\
\hline Module cost & $10 \$$ & $12 \$$ \\
\hline Networks & private/public & public \\
\hline Subscription cost & $\begin{array}{c}\text { free/below } 1 \$ \\
\text { per device/month }\end{array}$ & $1-2 \$$ per device/month \\
\hline Security & AES - 128 & 3GPP (128 - 256 bit) \\
\hline
\end{tabular}

${ }^{1}$ The maximum size of a Packet Data Convergence Protocol Service Data Unit (PDCP SDU) for NB-IoT.

communication technologies. Namely, the default LoRaWAN channels are located in the $868 \mathrm{MHz}$ band imposing DC restrictions of $1 \%[10]$ and allowing a device to transmit only for $36 \mathrm{~s}$ in each hour. Another restriction is related to the maximum Effective Radiated Power (ERP), which cannot exceed $14 \mathrm{dBm}$. However, this, along with the lack of signalling due to use of ALOHA-based channel access, has a positive effect on energy consumption.

The extended coverage of LoRaWAN is ensured by the use of Chirp Spread Spectrum (CSS) LoRa modulation. The LoRa modulation spreads narrowband signal into the 125 or $250 \mathrm{kHz}$ wide band. This increases resilience to narrowband interference and allows to operate under the noise level [8], [11]. Furthermore, LoRa modulation enables to change the signal spreading characteristics in terms of its slope and symbol duration. This is done through changing the Spreading Factors (SFs) parameter within the range of 7-12 (in EU) [10]. The higher SFs, resulting in each symbol gaining more energy, enable to extend the communication range [11]. High SFs also prolong the symbol transmission time, which increases the overall Time on Air (ToA) of a packet. This is also reflected in the maximal message size, which is 242 B for SF7, but only $51 \mathrm{~B}$ in the case of SF12.

From the communication perspective, a LoRaWAN enddevice (ED) utilizes ALOHA-like channel access. The ED may initiate communication at any time in one out of 16 available channels (three of them being mandatory) [10]. A gateway (GW) can initiate downlink transmission after a prespecified delay (typically $1 \mathrm{~s}$ ) measured from the end of the uplink transmission. In this phase, the ED opens Recieve Window 1 (RW 1) configuring the same channel and, typically, 
the same SF as were used for the uplink. If there is no downlink received in the first window, ED opens RW 2 after $1 \mathrm{~s}$ from the beginning of RW 1 . The RW 2 always uses predefined SF and channel (in EU typically $869.5 \mathrm{MHz}$ with SF12) [10]. The LoRaWAN EDs are subdivided into three classes. The support of class A is obligatory for all EDs and has been described above. Class B implies the presence of additional periodic RWs, and Class $\mathrm{C}$ requires a device to stay in reception all the time it is not transmitting. Apparently, changing the class of the ED from A towards C causes an increase in energy consumption.

\section{NB-IoT and LoRaWAN multi-RAT}

From Table I and the discussion above, one can see the key differences between the two LPWAN RATs. LoRaWAN EDs can operate with lower energy consumption, but are under stringent operation restrictions, affecting their throughput and not enabling for any QoS guarantees for delivery of their data. In contrast to them, the NB-IoT devices are less restricted for channel access and feature acknowledged and interference-free transmission, but present higher energy consumption.

Another important aspect is the monetary costs. The LoRaWAN networks can be deployed as private (i.e., owned by a user and targeted for specific application) or public (i.e., providing connectivity service to third-party users). The LoRaWAN equipment is commercially-available, allowing virtually anyone to deploy a network for under 1000 EUR. The NB-IoT operates in the licensed bands, requires costly equipment and thus is primarily deployed by telecom operators. Therefore, to use it, a contract with an operator is required.

As one can see, the combination of these two RATs enables: (i) reducing the costs, energy consumption, and latency whilst supporting reliable connectivity when NB-IoT is used as a back-off technology for a private LoRaWAN, (ii) increasing data transfer reliability and throughput when using two technologies simultaneously, and (iii) temporarily increase the throughput for specific operations (e.g., firmware upgrade) by switching to NB-IoT.

\section{IMPLEMENTATION OF LIVE MR-MMTC DEMONSTRATOR}

As discussed in our previous work [12], several approaches for multi-RAT implementation do exist. To build upon our previous expertise, the presented device within the scope of this paper stands for the fine-tuned hand-held battery-powered multi-RAT device enabling the sequential operation of RATs.

\section{A. Demonstrator Design}

The designed prototype is powered by the 32-bit microcontroller (MCU) STM32L152RDT6 capable of managing sequential communication for both LoRaWAN and NB-IoT modules. The device with overall dimensions $90 \times 117 \times 30 \mathrm{~mm}$ is designed as a hand-held tester. Note that the device is designed to support operation over only one RAT at a time and thus the positions of the antennas are not optimized to minimize the inter-RAT interferences.

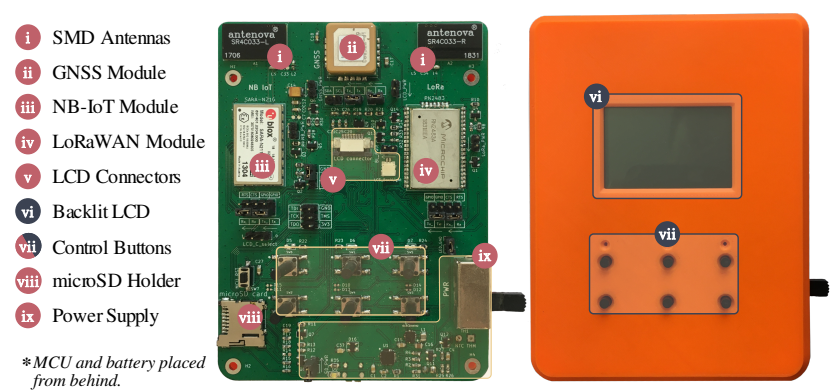

Fig. 1. Multi-RAT tester for NB-IoT \& LoRaWAN performance evaluation.

The key components of the demonstrator are highlighted in Fig. 1. The MCU is placed on the backside of the Printed Circuit Board (PCB). The radio signals are conveyed via (i) two surface mounted antennas SR4C033 from Antenova both having sufficient gain in $868 \mathrm{MHz}$ and $800 \mathrm{MHz}$ frequency band for LoRaWAN and NB-IoT modules, respectively. For precise localization, (ii) u-Blox SAM-M8Q GNSS module with an integrated antenna is used. The NB-IoT connectivity is provided by (iii) SARA-N211 radio module capable of operating in B8 and B20 frequency bands implementing the Rel. 13 of the 3GPP standard (i.e., as NB1 device). The Subscriber Identity Module (SIM) card holder for NB-IoT resides on the back face of the circuit board. The LoRAWAN connectivity is provided by (iv) RN2483 module. Both RAT modules are interfaced to the MCU through individual Universal Synchronous/Asynchronous Receiver and Transmitter (USART) interfaces.

The user interface for an operator is provided by the (vi) display unit interfaced to the MCU via Inter-integrated Circuit $\left(\mathrm{I}^{2} \mathrm{C}\right)$ bus. The navigation in the system interface is possible via the (vii) control buttons with integrated LEDs. The device also possesses other features like storing measured data to a (viii) micro SD card for subsequent evaluation. To enable the mobility of the tester, the device is fitted with a $3500 \mathrm{mAh} \mathrm{Li-}$ Pol battery with (ix) respective battery management circuitry. To handle the high current consumption of SARA-N211 in transmit, the tester is equipped with buck/boost converter ensuring constant core voltage level of $3.3 \mathrm{~V}$.

\section{B. Evaluation Procedure}

To test the developed prototype and to gain some insights into the potential of NB-IoT-LoRaWAN multi-RAT operation in the context of a practical Smart City application, we evaluated its performance with respect to one practical use case - the public transport vehicles tracking. The measurements were performed in 74 test locations around the city of Brno, the Czech Republic, on the public transport stops along the two traces: east-west and north-south direction, which are depicted in Figs. 2 and 3, respectively.

During our experiment, all measurements followed the same pattern. The prototype was transferred to the desired test location, from where it was sending 10 messages with a period of $30 \mathrm{~s}$ with either 50 or 200 bytes application-layer payload each [13]. It is worth mentioning that the uplink messages 


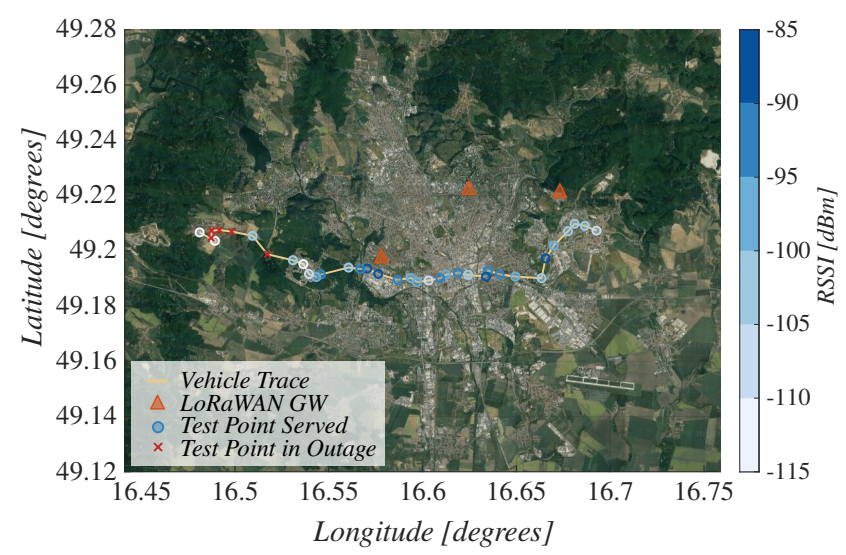

Fig. 2. Measurement locations in East-West direction.

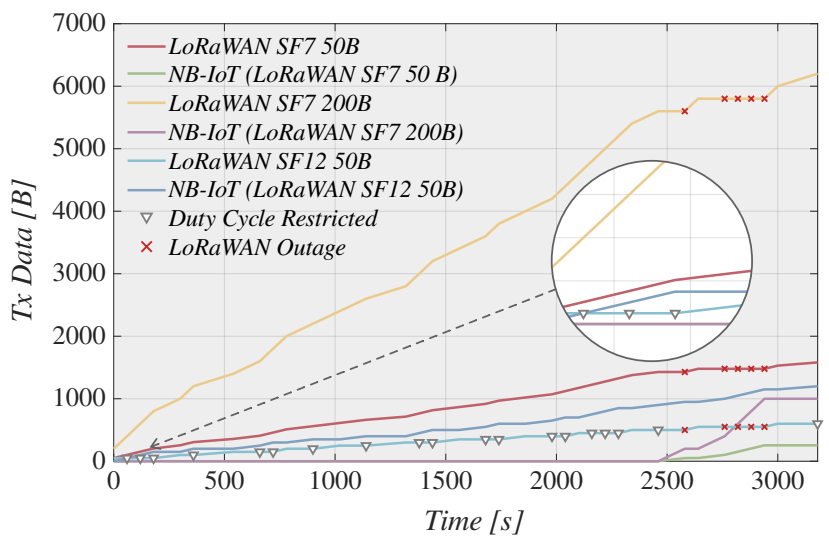

Fig. 4. Cumulative throughput vs time for loc. in East-West direction.

were sent to the LoRaWAN GWs without any subsequent acknowledgement in downlink using SF7 or SF12. In case the data or their part could not be transmitted over LoRaWAN due to DC limitations or other issues, they were handled to NB-IoT transceiver for transmission. Switching between RATs during such occasions was performed manually without the usage of any autonomous optimization method. When all the measurements were finished, the results were downloaded from the IoT data management platforms (located at Brno University of Technology) for further analysis.

For our measurements, we have used the communication infrastructure of České Radiokomunikace (CRa) LoRaWAN and Vodafone Czech Republic NB-IoT networks. For NBIoT, the Vodafone utilizes a guard band of the LTE spectrum in B20 (800 MHz). Following the EU regulations, the CRa LoRaWAN network was configured to run in $868 \mathrm{MHz}$ with a $1 \%$ DC limitation. Note, that to mimic the operation of a private multi-GW LoRaWAN network (e.g., deployed by the traffic management authorities), we considered LoRaWAN data delivered through only three CRa GWs located in the central area of the city of Brno.

\section{Discussion of results}

From the results presented in Figs. 2 and 3 one can see that only from 6 out of 74 locations points, which were tested, no

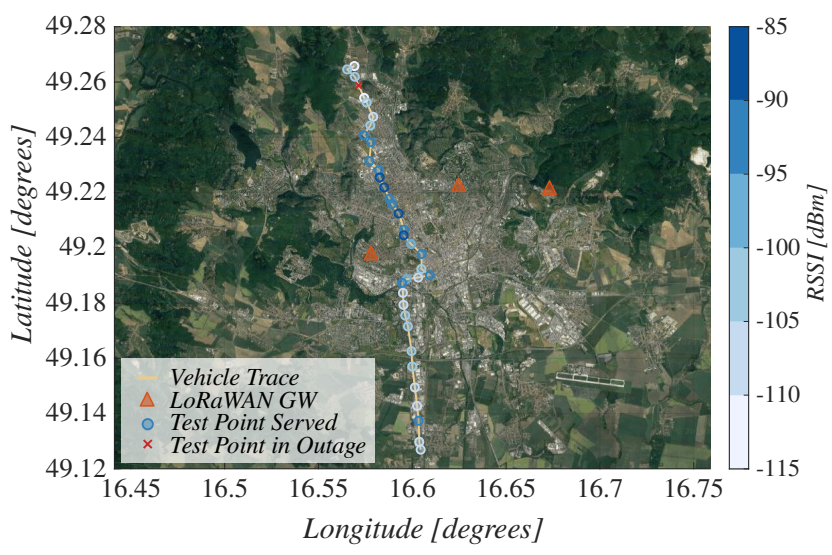

Fig. 3. Measurement locations in North-South direction.

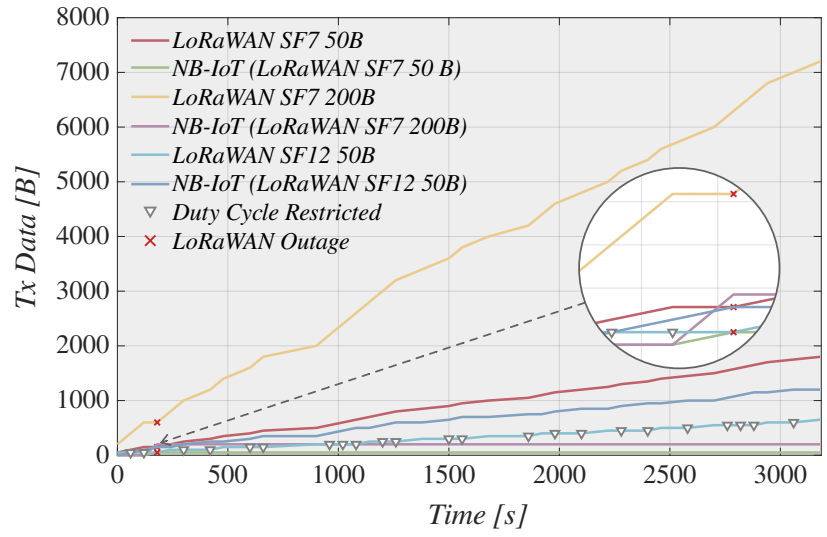

Fig. 5. Cumulative throughput vs time for loc. in North-South direction.

data transfer over LoRaWAN was possible. These results show that Brno as a mid-size city with approx. population of 400000 can be covered with $90 \%$ probability using LoRaWAN technology with only three GWs ${ }^{1}$. This observation is especially important for small technology integrators as it shows that they can deploy a city-scale network without huge investments. Another interesting observation is that during measurements, the minimum Received Signal Strength Indicator (RSSI) level of LoRaWAN signals was above $-115 \mathrm{dBm}$, providing an $8 \mathrm{~dB}$ margin to the sensitivity of a LoRaWAN receiver for a signal with SF7.

Figs. 4 and 5 provide further insight into the distribution of the traffic between the technologies for different packet payload size and SF configuration for LoRaWAN transmissions. The charts illustrate the cumulative data transferred through each RAT as a function of the time elapsed since the start of the experiment. Note, that in the legend for NB-IoT in brackets is listed the payload size and the SF configuration for LoRaWAN. Also keep in mind that in the case of licenseexempt communication technologies, one has to consider not just the outage, but also the DC limitations. Both these cases are marked explicitly in Figs. 4 and 5.

\footnotetext{
${ }^{1}$ The assumption is valid only for the city of Brno, other cities of similar size and population but with different terrain and weather conditions could show different results.
} 
As one can see, for the messages conveyed using SF7, the DC of $1 \%$ is not violated even with application payload size of $200 \mathrm{~B}$. On the other hand, for $50 \mathrm{~B}$ messages transmitted with SF12, the DC limitation prevented data transmission $46 \%$ of the time on average. This demonstrates the importance of the LoRaWAN SF optimization to maximize not only the ED's lifetime but also to magnify the application throughput.

As one can see from Fig. 4 (2500 to 3000 seconds region) and Fig. 5 (operation for SF12), in both observed situations when data transfers were impossible (i.e., an outage and a DC restriction), the second RAT successfully backed-up LoRaWAN and allowed data delivery. This demonstrates that the NB-IoT coverage is omnipresent (the average value of measured Reference Signal Received Power (RSRP) for NBIoT across all test locations was $-85 \mathrm{dBm}$, meaning that the channel conditions are rather comfortable).

In addition to confirming the correct operation of the developed multi-RAT prototype, our results show that for the targeted applications of public transport vehicles tracking, the multi-RAT approach enables to transfer up to $80-92 \%$ of the traffic in the unlicensed bands, thus enabling cost reductions. The licensed bands and energy-hungry NB-IoT technology may be used only when really needed - in case of outages or once reaching the DC limit.

\section{CONCLUSIONS}

In this paper, we reported on the design of a multi-RAT device utilizing two complementary LPWAN technologies. At the same time, the primary one (LoRaWAN) operates in the license-exempt frequency band, and the backup one (NBIoT) uses the licensed part of the frequency spectrum. The choice of LoRaWAN as the main communication enabler is based on the ability to construct own private network with significantly lower operating expense. The functionality of the constructed prototype as well as the verification of the real-life performance in the Smart City scenario for tracking the position of the public transport vehicles were successfully handled in the course of the proof-of-concept assessment.

The results gathered from an extensive measurement campaign in the mid-size European city (Brno, Czech Republic) highlight the pros and cons of the selected technologies. By using the LoRaWAN for data transmissions at each bus / tram stop as the primary connectivity option, it was possible to serve at best $92 \%$ of measured locations. The data transmissions in the remaining locations were then managed using NB-IoT technology. Nevertheless, the data we got revealed that the connectivity (communication range) is not an issue. The key challenge when using the technology working in the licenseexempt spectrum is to keep the spectrum utilization bellow $1 \%$ DC. Moreover, as it can be seen from the Fig. 4 and Fig. 5, configuring the end-device with the optimal spreading factor is the key for achieving sufficient ToA.

Another challenge comes with the question of optimal and cost-effective RAT switching. For Capital Expenditures (CAPEX), LoRaWAN suffers from a need for initial costs of infrastructure deployment whereas, for NB-IoT, there is the only cost per module. On the other hand, in case of Operating Expense (OPEX), LoRaWAN private networks are free for a subscription. In contrary, NB-IoT is conditioned by subscription with a fee being between $1-2 \$$ per $100 \mathrm{kB}$ of transmitted data. With above mentioned in mind, an effective way of optimized switching between mentioned RATs considering technology restrictions and subscription cost should be implemented in future work (e.g., by simple machine learning approach).

Considering the acquired data and the overall functionality of developed prototype, many of use-cases coming from the Smart City domain i.e., mMTC scenarios can benefit from the opportunity to use complementary communication technologies while transmitting data. As the functionality of having access to the core network for the non-3GPP technologies has been officially enabled through the N3IWF feature, the seamless combination of communication technologies will play a crucial role in the B5G networks.

\section{ACKNOWLEDGMENT}

For the research, the infrastructure of the SIX Center was used. The work of K. Mikhaylov has been supported by Academy of Finland 6G Flagship (grant 318927). The described research was financed by the Technology Agency of the Czech Republic project No. TN01000007.

\section{REFERENCES}

[1] X. Chen and T. Han, "Disruptive Technology Forecasting Based on Gartner Hype Cycle," in Proc. IEEE Techn. Eng. Management Conf., June 2019, pp. 1-6.

[2] M. Z. Chowdhury, M. Shahjalal, S. Ahmed, and Y. M. Jang, "6G Wireless Communication Systems: Applications, Requirements, Technologies, Challenges, and Research Directions," arXiv preprint:1909.11315, 2019.

[3] R. Sanchez-Iborra, J. Santa, J. Gallego-Madrid, S. Covaci, and A. Skarmeta, "Empowering the Internet of Vehicles with Multi-RAT 5G Network Slicing," Sensors, vol. 19, no. 14, pp. 1-16, 2019.

[4] H. Tabassum, M. Salehi, and E. Hossain, "Mobility-Aware Analysis of 5G and B5G Cellular Networks: A Tutorial," arXiv preprint:1805.02719, 2018.

[5] K. Mikhaylov, J. Petäjäjärvi, M. Mäkeläinen, A. Paatelma, and T. Hänninen, "Extensible Modular Wireless Sensor and Actuator Network and IoT Platform with Plug\&Play Module Connection," in Proc. 14th Int. Conf. Inf. Proces. in Sensor Netw., April 2015, pp. 386-387.

[6] O. Galinina, A. Pyattaev, S. Andreev, M. Dohler, and Y. Koucheryavy, "5G multi-RAT LTE-WiFi Ultra-dense Small Cells: Performance Dynamics, Architecture, and Trends," IEEE J. Selected Areas in Commun., vol. 33, no. 6, pp. 1224-1240, 2015.

[7] A. Ometov, "System-level Analysis of IEEE 802.11ah Technology for Unsaturated MTC Traffic," Int. J. Sensor Netw., vol. 26, no. 4, pp. 269282,2018

[8] "LoRaWAN and NB-IoT: Competitors or Complementary," $A B I R e$ search, 2019.

[9] R. Mozny, P. Masek, M. Stusek, K. Zeman, A. Ometov, and J. Hosek, "On the Performance of Narrow-band Internet of Things (NB-IoT) for Delay-tolerant Services," in Proc. 42nd Int. Conf. Telecommun. and Signal Proces., July 2019, pp. 637-642.

[10] N. Sornin, "LoRaWAN® Specification v1.1," LoRa Alliance, 2017.

[11] B. Reynders, W. Meert, and S. Pollin, "Range and Coexistence Analysis of Long Range Unlicensed Communication," in Proc. 23rd Int. Conf. on Telecommun., May 2016, pp. 1-6.

[12] K. Mikhaylov et al., "Multi-RAT LPWAN in Smart Cities: Trial of LoRaWAN and NB-IoT Integration," May 2018, pp. 1-6.

[13] “3GPP Low Power Wide Area Technologies," GSMA, White Paper, May 2018. 